\title{
Characterization of Periodic, Quasiperiodic, and Chaotic States in Nonpremixed Biodiesel/Air Jet Flames
}

\author{
Jianxin Xu, ${ }^{1}$ Hua Wang, ${ }^{1}$ and Hui Fang ${ }^{2}$ \\ ${ }^{1}$ Engineering Research Center of Metallurgical Energy Conservation and Emission Reduction, Ministry of \\ Education, Kunming University of Science and Technology, Yunnan 65009, China \\ ${ }^{2}$ Department of Applied Mathematics, Kunming University of Science and Technology, \\ Yunnan 650093, China
}

Correspondence should be addressed to Hua Wang, wanghuaheat@hotmail.com

Received 8 April 2011; Revised 31 July 2011; Accepted 22 August 2011

Academic Editor: Christos H. Skiadas

Copyright (C) 2011 Jianxin Xu et al. This is an open access article distributed under the Creative Commons Attribution License, which permits unrestricted use, distribution, and reproduction in any medium, provided the original work is properly cited.

Characterization for nonpremixed biodiesel/air jet flames instability is investigated by the $0-1$ test for chaos and recurrence plots. Test conditions involve biodiesel from Jatropha curcas. Lfueled flames have inlet oil pressure of $0.2-0.6 \mathrm{MPa}$, fuel flow rates $(Q 1)$ of $15-30 \mathrm{~kg} / \mathrm{h}$, and combustion air flow rate $(Q 2)$ of $150-750 \mathrm{~m}^{3} / \mathrm{h}$. This method is based on image analysis and nonlinear dynamics. Structures of flame are analyzed using an image analysis technique to extract position series which are representative of the relative change in temperature of combustion chamber. Compared with the method of maximum Lyapunov exponent, the $0-1$ test succeeds in detecting the presence of regular and chaotic components in flame position series. Periodic and quasiperiodic characteristics are obtained by the Poincaré sections. A common characteristic of regular nonpremixed flame tip position series is detected by recurrence plots. Experimental results show that these flame oscillations follow a route to chaos via periodic and quasiperiodic states.

\section{Introduction}

Unsteady motions of the nonpremixed biodiesel-fueled flames in combustion chamber are investigated from the viewpoint of the deterministic chaos theory. The periodic and chaotic motions in flame dynamics that can be observed as a result of flame instabilities are of great importance to present-day combustion physics and thermal fluid science research [1]; they also are significant in problems that involve combustion instabilities within engines, boilers, and furnaces. When combustion instabilities occur, the combustion process is characterized by the coupling of various nonlinear phenomena, leading to the formation of either a limit 
cycle or chaos. Generally, there are three ways leading to chaos: period-doubling bifurcation, intermittent chaos, and quasiperiodic into chaotic. In high-dimensional case, it appears much more complexity in chaotic development model. Fichera et al. [2] have verified that combustion instability is governed by chaos, using the nonlinear time series analysis techniques, stable combustion system means that the system is pushed towards chaotic regimes.

In our experiments, the fuel flow rate and combustion air flow are varied, and the proportion between the fuel flow rate and combustion air flow is investigated. The main aim of the present study is to characterize combustion instabilities by nonlinear time series analytical methods based on the deterministic chaos theory, including spectrum analysis, strange attractor, correlation dimension, the surrogate data, and maximum Lyapunov exponent (MLE) methods [1,3-6]. Recently, a new test called "the 0-1 test" has been proposed to detect the presence of chaos in time series [7-9]. Unlike the above methods, the 01 test has the advantage of being easy to implement and does not need the underlying equations, the reconstruction of the phase space, or the dimension of the actual system. The input is the time series data, and the output is 0 or 1 depending on whether the dynamics is nonchaotic or chaotic. It has been applied successfully on theoretical time series, both noiseless and noisy, from various dynamical systems (van der Pol, Kortwegde Vries, Lorenz, the logistic map), and on experimental data [10, 11]. In this work, we verify the validity of the $0-1$ test for chaos by using experimental data of nonpremixed flame oscillation.

Position series are extracted from flame tip structures using a novel technique based on image analysis procedures, then the 0-1 test for chaos and the MLE method are employed to detect the presence of chaos. Some periodic or quasiperiodic components are found in position series by the $0-1$ test for chaos and Poincaré map and could be characterized by means of recurrence plots.

\section{Experiments and Methodology}

\subsection{Experimental Setup}

The experimental setup is installed on a scaled combustion chamber at atmospheric pressure, equipped with quartz windows to have direct optical access to the flame. The spatial structure and time evolution of the flame are recorded through a high-frequency and intensified camera. The fuel is biodiesel from Jatropha curcas. L. Test conditions involve inlet oil pressure of $0.2-0.6 \mathrm{MPa}$, fuel flow rates $(Q 1)$ of $15-30 \mathrm{~kg} / \mathrm{h}$, and combustion air flow rate $(Q 2)$ of $150-$ $750 \mathrm{~m}^{3} / \mathrm{h}$. Liquefied petroleum gas is used in the ignition system. Flame lengths are limited to $300-600 \mathrm{~mm}$, as seen in Figure 1.

\subsection{Methods of Analysis}

\subsubsection{Extraction of Position Series from the Flame Structure}

With the development of digital image processing techniques, on-line continuous monitoring of the flame is becoming possible. Conventional CCD cameras and flame grabbers are required to collect the flame image. The strength of using video in the image detection is the ability to monitor large and open spaces. A digital color camera is installed to capture 


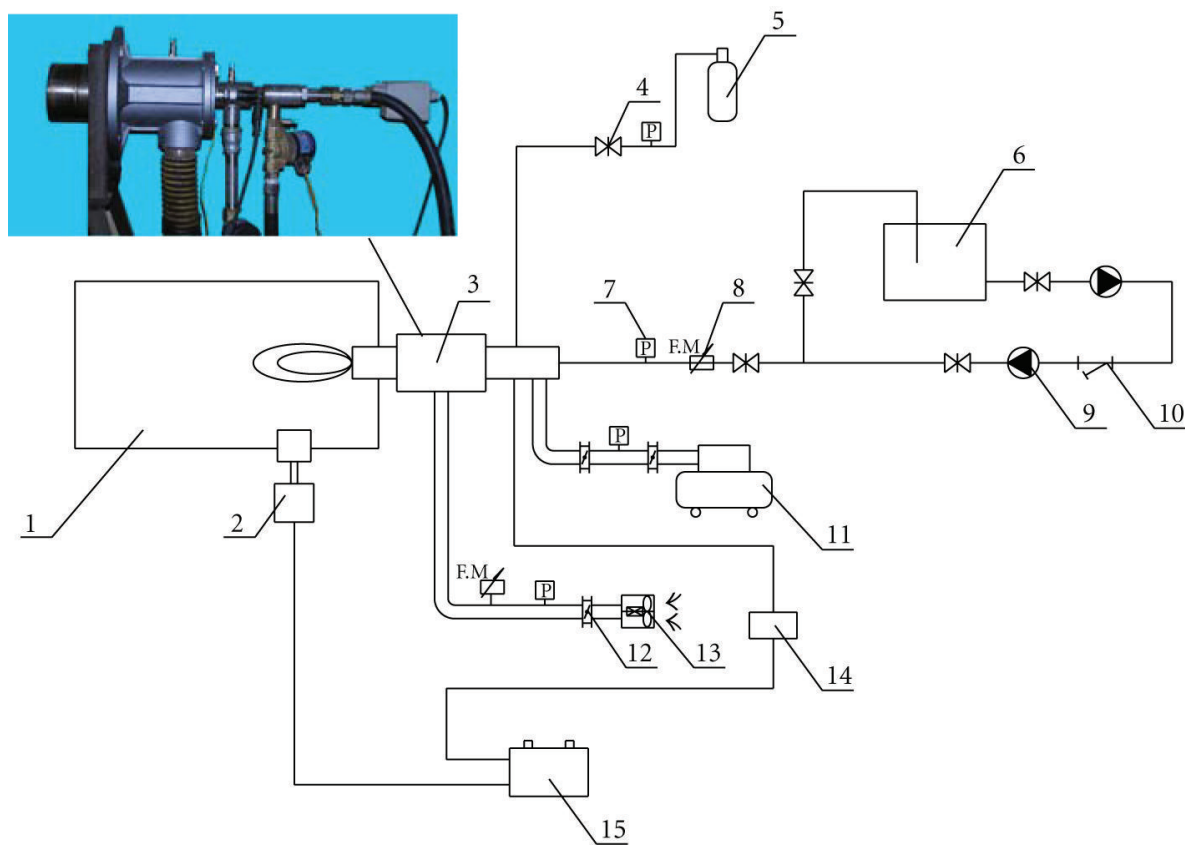

Figure 1: Experimental setup: 1: combustion chamber; 2: camera; 3: burner; 4: gate valve; 5: gas tank; 6: oil tank; 7: pressure gauge; 8: flow meter; 9: oil pump; 10: filter; 11: air compressor; 12: air valve; 13: blower; 14: igniter; 15: power supply.

the images of flames in the furnace. The camera with the specifications of $350 \times 550$ pixels is capable of capturing flame images at 25 frames per second. The output signal of the camera is sent to a computer. To protect the CCD camera from damage caused by high temperature and keep image qualities from dust, an air-cooling device is used. A set of optical filters is connected to the front of the camera to gather more robust flame images, prevent the camera from saturation, and distinguish the colors in the inner and the outer flame parts. Such optical filters make sure that the system can capture flame images over the visible spectral band [12]. It is noted that the optimal camera settings are selected and fixed so that the images grabbed in oil-fired combustion processes can possess the maximum information. All images under the normal operation are recorded realtime and are retained in a data storage system, providing a wealth of data for analysis. In our experiments, the actual flame direction is from right to left, in order to give readers a better understanding of this method of extracting position series, Figure 2(a) shows one of sequential images in the normal condition, which is flipped horizontally. However, because all position series are obtained along the growth direction of the flame, which is not changed, the interval between the images is one minute.

To get characterization for nonpremixed biodiesel/air jet flames instability, one can extract position series from the flame tip structures in reverberatory furnace combustion chamber using the following method.

Step 1. A camera is used to gain a frame per minute and take 20 images in the experiment. The following image analysis procedure is adopted [13]. Color pictures of the flame tip structures are processed to produce grey scale images in which the lowest color (black) and the highest 


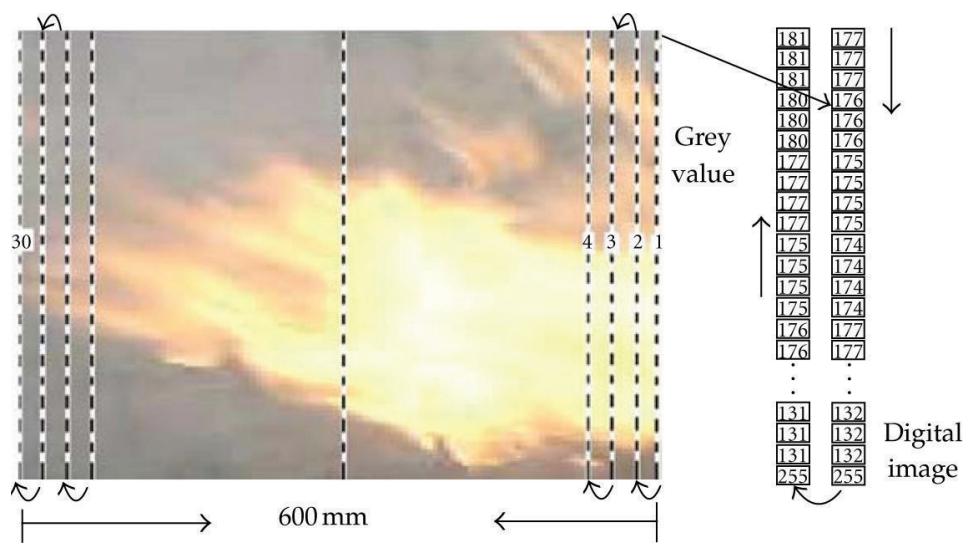

(a)

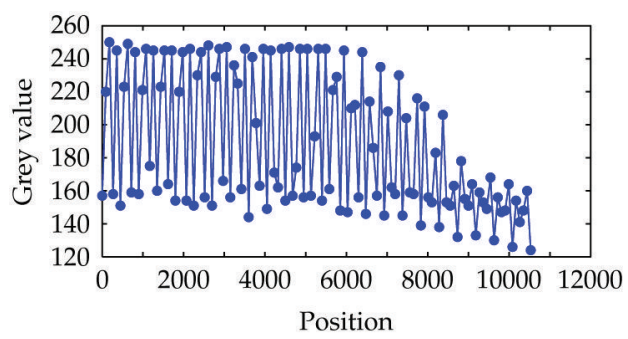

(b)

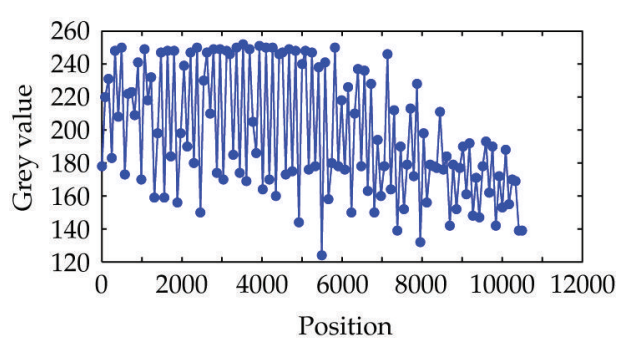

(c)

Figure 2: (a) shows the method used to extract position series from the flame tip structures. The blue dots show the resampling executed to avoid oversampling: every 89 points in (b) and 82 points in (c).

(white) are represented by the color codes 0 and 255 and can be considered representative of the relative change in temperature. Size of the image is $350 \times 550$ (pixels), and this image is traced 30 transverses with equal distance. Figure 2 shows that a number of transverses passing through the grey scale of images are traced, then position series are produced whose values range between 0 and 255 in grey intensity values, with 10500 points $(350 \times 30)$, where every point represents a position. Because of spatial continuity of the flame tip structures, each space position series has been connected to the successive to obtain a single position series for each image. Since space position series is a set of discrete data sampled in sequence, we may deal with it in a similar way as time series. In the sequel, to avoid confusion, we still call position series time series.

Step 2. Obtain the median $k$ of $k_{c}$ for each space position series by $0-1$ test for chaos. Since the dynamical behaviors of the flame space position series could be characterized by $k_{c}$ patterns, and $k_{c}$ evolves with time (as showed in Figure 10), one can get characterization for nonpremixed biodiesel/air jet flames instability. In this paper, odd and even numbers sequences are both applied for the nonlinear dynamical analysis of position series of nonpremixed biodiesel flames. Since the oversampled time series might cause the 0-1 test to fail, it is important to obtain the optimal sampling time. A good choice of the sampling time can often be obtained by using the first minimum of the mutual information to avoid oversampling [14]. 
In this work, we study spatial patterns rather than time series, through these spatial patterns, some position series are obtained by an image analysis technology, and then used to detect chaos of these spatial patterns by the $0-1$ test for chaos. The position series in Figures 2(b) and 2(c) do not appear to be stationary. Actually, in our works, the experimental data is obtained after a long time, when the average temperature in combustion chamber has little changes. And all the position series obtained by this method [13] are almost not stationary including the position series from the magma mixing structures. Poli and Perugini [13] use these nonstationary position series to reconstruct the attractors and to calculate the fractal dimension of the attractors. However, these position series of spatial patterns could be analyzed directly using chaotic theory.

In fact, the features of the flame, such as geometrical and luminous profiles, can provide important and rich information on the quality of the flame to measure the performance of the combustion process. Some CCD-image-based methods were used to construct the flame temperature distribution instead of laser-based methods because of their advantages of simplicity and low cost. In our work, the original color encoding is RGB. It can be reshaped into a sequence of column vectors using MATLAB program; the color RGB images are recorded by the camera experimentally. The intensities or colors recorded by a camera were helpful in achieving a visual understanding of various flame structures. Progress has also been made in flame color analysis which is able to evaluate combustion temperature by correlating the RGB signals of digital flame images. MATLAB command, rgb2gray (1992-2004 The MathWorks, Inc.) is utilized. The rgb2gray converts RGB values to gray scale values by forming a weighted sum of the $R, G$, and $B$ components:

$$
\text { Gray }=0.2989 \times R+0.5870 \times G+0.1140 \times B \text {. }
$$

This is the observable and not the average grey.

\subsubsection{Detection of Chaos and Recurrence Analysis}

In this paper, both the classical method (MLE) and the 0-1 test for chaos are employed to detect the chaos in flame position series. The Lyapunov exponents are also calculated for the position series using the algorithm of Rosenstein [15] (as seen in Figure 10). The time delay and embedding dimension are computed using the Visual Recurrence Analysis (VRA) [16]. Mutual information function can be used to determine the optimal value of the time delay for the state space reconstruction. The embedding dimension is computed using the technique of false nearest neighbors (FNNs) [17], then the phase space is reconstructed, as seen in Figure 9.

Given an observation $\phi(j)$ for $j=1, \ldots, N, \phi(j)$ represents a one-dimensional observable data set. For $c \in(0, \pi)$, we compute the translation variables:

$$
p_{c}(n)=\sum_{j=1}^{n} \phi(j) \cos j c, \quad q_{c}(n)=\sum_{j=1}^{n} \phi(j) \sin j c, \quad n=1,2,3, \ldots, N .
$$


To determine the growth of $p_{c}$ and $q_{c}$, it is convenient to look at the mean square displacement (MSD), defined as

$$
M_{c}(n)=\lim _{N \rightarrow \infty} \frac{1}{N} \sum_{j=1}^{N}\left[\left(p_{c}(j+n)-p_{c}(j)\right)^{2}+\left(q_{c}(j+n)-q_{c}(j)\right)^{2}\right] .
$$

Note that this definition requires $n \ll N$. In practice we find that $n_{\text {cut }}=N / 10$ yields good results. The test for chaos is based on the growth rate of $M_{c}(n)$ as a function of $n$. The next step is to estimate the asymptotic growth rate $K_{c}$. We have tried out two different methods: a regression method and a correlation method.

\section{Regression Method}

We have

$$
k_{c}=\lim _{N \rightarrow \infty} \frac{\log M_{c}(n)}{\log n}
$$

Numerically, $k_{c}$ is determined by fitting a straight line to the graph of $\log M_{c}(n)$ versus $\log n$ through minimizing the absolute deviation.

\section{Correlation Method}

We now present an alternative method for determining $k_{c}$ from the mean square displacement. Form the vectors $\xi=\left(1,2,3, \ldots, n_{\text {cut }}\right)$ and $\Delta=\left(M_{c}(1), M_{c}(2), M_{c}(3), \ldots, M_{c}\left(n_{\text {cut }}\right)\right)$. Given vectors $x, y$ of length $q$, we define covariance and variance in the usual way:

$$
\begin{gathered}
\operatorname{cov}(x, y)=\frac{1}{q} \sum_{j=1}^{q}(x(j)-\bar{x})(y(j)-\bar{y}), \quad \text { where } \bar{x}=\frac{1}{q} \sum_{j=1}^{q} x(j), \\
\operatorname{var}(x)=\operatorname{cov}(x, x) .
\end{gathered}
$$

Now define the correlation coefficient

$$
k_{c}=\operatorname{corr}(\xi, \Delta)=\frac{\operatorname{cov}(\xi, \Delta)}{\sqrt{\operatorname{var}(\xi) \operatorname{var}(\Delta)}} \in[-1,1]
$$

$k_{c}=0$ for regular dynamics and $k_{c}=1$ for chaotic dynamics.

Unlike the classic method of calculating MLE, the 0-1 test does not need any information about the dynamics. The $0-1$ test is a binary test in the sense that it can only distinguish between nonchaotic and chaotic dynamics. In this approach, the regular and chaotic motions can be decided by calculating the parameter $k_{c}$ approaching asymptotically to zero or one. $k_{c}$ is defined as the asymptotic growth rate of the mean square displacement (MSD), and $k_{c}$ can be numerically determined by means of linear regression of $\log M(t)$ versus $\log t$ or correlation method $[8,9,14]$. As pointed out in [14], the median is robust against outliers associated with resonances. $k_{c}$ should be taken as the median rather than 
the mean. Another interesting feature of the 0-1 test for chaos is that the inspection of the dynamics of the $(p, q)$-trajectories provides a simple visual test of whether the underlying dynamics is chaotic or nonchaotic [18]. Furthermore, it is relatively easy to implement. The test only gives two possible results: close to 0 for a nonchaotic system and close to 1 for a chaotic system [11]. Our experimental study on the position series of flame tip structures verifies well with this method.

Recurrence plots are graphical devices specially suited to detect hidden dynamical patterns and nonlinearities in data. However, there are few programs available to apply such a methodology. In this work, one of the best free programs to apply nonlinear position series analysis, Visual Recurrence Analysis (VRA), is introduced to characterize periodic or quasiperiodic position series of nonpremixed flame tip. VRA calculates the (Euclidean) distances between all pairs of vectors and codes them as colors. After the distances between all vectors are calculated, they are mapped to colors from the predefined color map and are displayed as colored pixels in their corresponding places. A recurrence plot is essentially a graphical representation of a correlation integral [16]. See Figures 6 and 8.

By the $0-1$ test for chaos, it is found that regular and chaotic flame position series could be characterized by $k_{c}$ patterns and $(p, q)$-plot, and recurrence plots are used to further describe the different dynamical patterns and nonlinearities between the chaos and quasiperiod or periodic. The Poincare sections are obtained to analyze the different dynamical patterns and nonlinearities between the quasi-period and periodic, as seen in Figure 4. A common characteristic of quasiperiodic or periodic position series in nonpremixed flame tips is found by recurrence plots, as seen in Figure 6.

\section{Results and Discussion}

Nonlinear time series analysis is significant for gaining a comprehensive understanding of complex nonlinear phenomena, as mentioned in [2], whether or not unstable combustion is governed by chaos. If the nonlinear behaviors are chaotic, it means that the combustion system is stable, unstable otherwise. In this study, we obtain such a characterization of nonpremixed flame instabilities using the 0-1 test for chaos and recurrence plots. Furthermore, we find that the nonpremixed biodiesel/air jet flame instabilities follow a route to chaos via periodic and quasiperiodic states.

As pointed out in $[9,14]$, the dynamics of the translation components $(p, q)$ are corresponding to the underlying dynamics. Bounded trajectories are corresponding to periodic or quasiperiodic dynamics, and Brownian-like trajectories are corresponding to chaotic dynamics. In Figure 3, we give the $q$-p-plot using the method described in $[9,14]$.

In Figures 3(a), 3(b), and 3(c), the dynamics of the translation components $(p, q)$ of flame position series are bounded trajectories; however, how do we decide whether dynamics is periodic or quasiperiodic? The Poincaré sections are obtained from these flame position series, as is shown in Figure 4. For a periodic case, the Poincaré section is only one fixed point or a few discrete points in plane. For a quasiperiodic case, the Poincare section is a closed curve with points in plane. Figure 4(a) shows a few discrete points in plane, which indicates periodic dynamics. Because there are not enough data points, Figures $4(\mathrm{~b})$ and 4 (c) approximately show a closed curve with points in plane, which indicates quasiperiodic dynamics.

The median $k$ of $k_{c}$ values are close to 0 in the nonchaotic case and close to 1 in the chaotic case. Since the correlation method of the 0-1 test cannot detect resonances which 


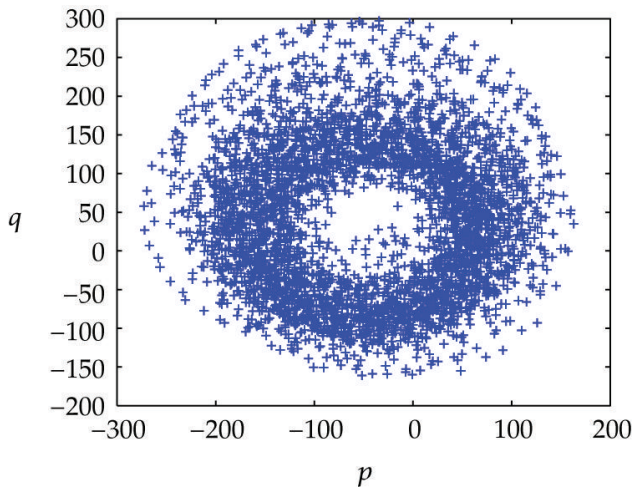

(a)

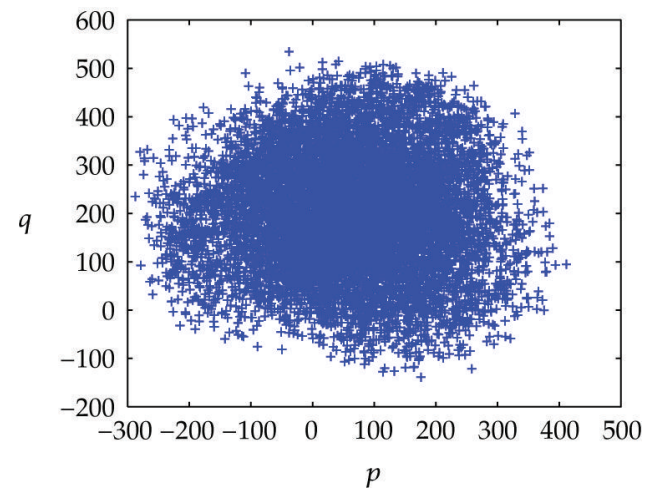

(c)

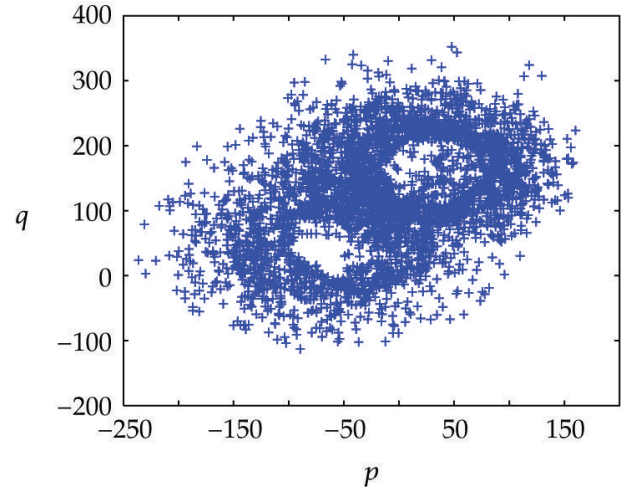

(b)

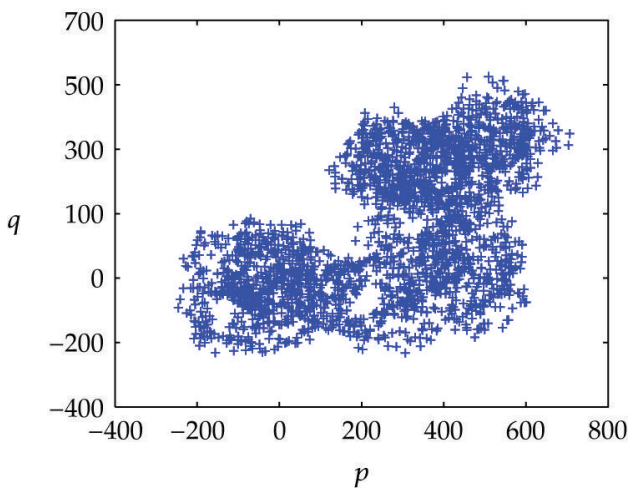

(d)

Figure 3: The dynamics of the translation components $(p, q)$ of flame position series. (a) Bounded trajectories are shown corresponding to periodic dynamics at $k=0.5046, Q 1=15 \mathrm{~kg} / \mathrm{h}$, and $Q 2=$ $150 \mathrm{~m}^{3} / \mathrm{h}$. (b) Bounded trajectories are shown corresponding to quasiperiodic dynamics at $k=0.6482$, $Q 1=15 \mathrm{~kg} / \mathrm{h}$, and $Q 2=200 \mathrm{~m}^{3} / \mathrm{h}$. (c) Bounded trajectories are shown corresponding to quasiperiodic dynamics at $k=0.7646, Q 1=20 \mathrm{~kg} / \mathrm{h}$, and $Q 2=260 \mathrm{~m}^{3} / \mathrm{h}$. (d) Brownian-like trajectories are shown corresponding to chaotic dynamics at $k=0.8081, Q 1=20 \mathrm{~kg} / \mathrm{h}$, and $Q 2=230 \mathrm{~m}^{3} / \mathrm{h}$.

are expected to occur in quasiperiodic data, one doubles check in Figures 5 and 7 using the version of the regression method described in [14].

In Figure 9, recurrence plots are used to show which vectors in the reconstructed space are close and far from each other. However, there are no similar or common characteristics for chaotic time series. So recurrence plots could not characterize the chaotic flame position series. Thus, for the periodic or quasiperiodic position series, a common characteristic is obtained in Figure 6, the time delay $(\tau)$ is determined by mutual information function, and the embedding dimension $(D)$ is computed using the technique of false nearest neighbors (FNNs) [17]. $\tau=6,7,6, D=9,9,9$ in periodic or quasiperiodic case; $\tau=5,5,7, D=$ 9, 10, 10 in chaotic case.

Although the value of $k$ rapidly varies with $c$ in Figure 5 as in Figure 7, the median $k$ of $k_{c}$ in Figures 5 and 7 is quite different. When the proportion between the fuel flow rate and combustion air flow is approximately controlled at $1: 12$, the combustion is relatively stable, and the positional series exhibit chaotic motion. 


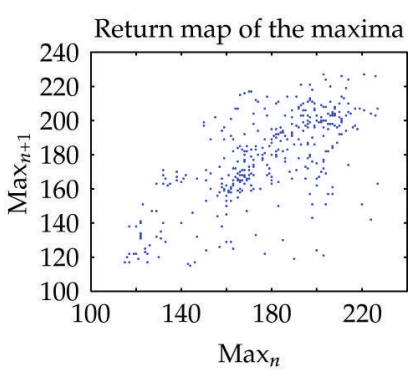

(a)

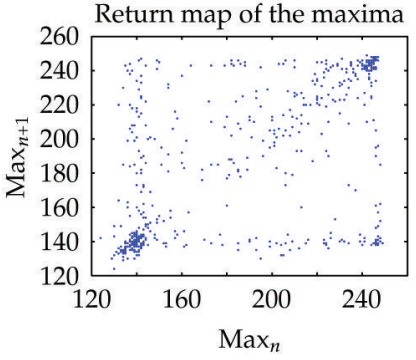

(b)

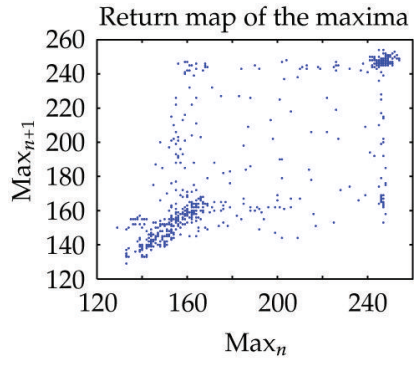

(c)

Figure 4: Poincaré sections from periodic or quasiperiodic position series: (a) $k=0.5046$ for periodic position series; (b) $k=0.6482$ for quasiperiodic position series; (c) $k=0.7646$ for quasiperiodic position series.

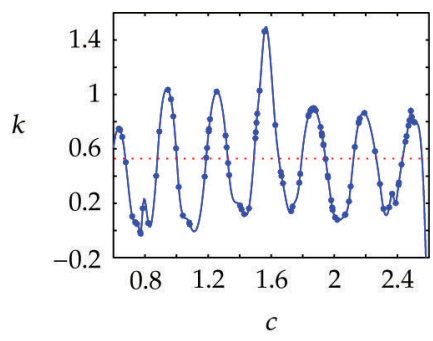

(a)

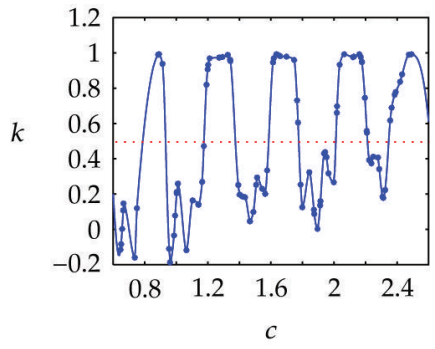

(d)

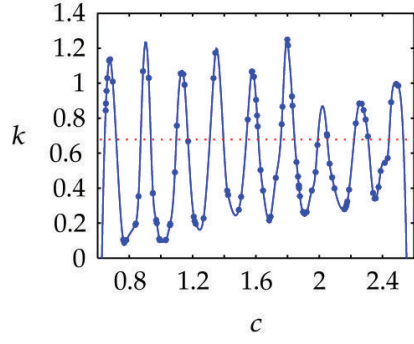

(b)

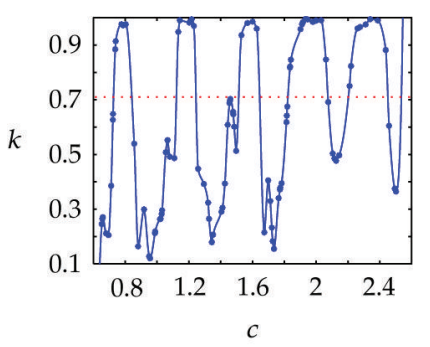

(e)

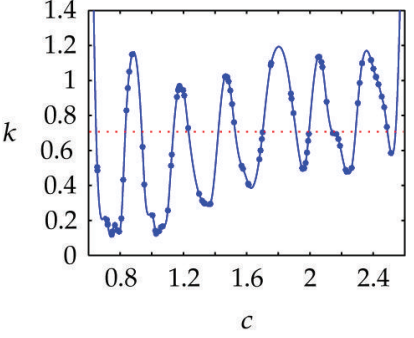

(c)

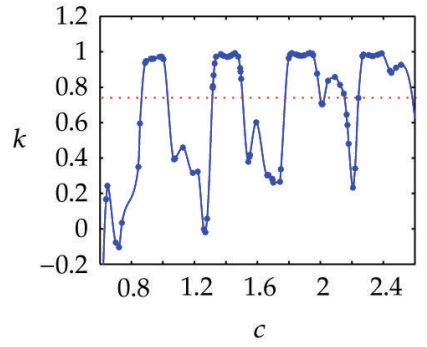

(f)

Figure 5: $k_{c}$ in the case of periodic or quasiperiodic position series using the regression method (top) and correlation method (bottom): (a) $k=0.5628$; (b) $k=0.6542$; (c) $k=0.7126$; (d) $k=0.5046, Q 1=15 \mathrm{~kg} / \mathrm{h}$, $Q 2=150 \mathrm{~m}^{3} / \mathrm{h}$ for periodic position series; (e) $k=0.7194, Q 1=18 \mathrm{~kg} / \mathrm{h}, Q 2=250 \mathrm{~m}^{3} / \mathrm{h}$ for quasiperiodic time series; (f) $k=0.7646, Q 1=20 \mathrm{~kg} / \mathrm{h}, Q 2=260 \mathrm{~m}^{3} / \mathrm{h}$ for quasiperiodic position series. Red dashed lines in (a)-(f) stand for the median $k$ of $k_{c}$.

In Figure 6, red, green, and blue regions are distributed with distinct boundaries. In Figure 8 , red, green, and blue regions are distributed with vague boundaries.

In Figures 9 and 10, we take the computational results of a chaotic time series for an example. Both the MLE and the 0-1 test succeed in detecting the presence of chaos in nonpremixed flame position series. For all of position series, there are only two cases: the maximum Lyapunov exponent is positive suggesting the dynamic is chaotic, nonchaotic otherwise. However, the $0-1$ test for chaos to characterize flame instability is more rapid than the classical method of calculating MLE. 


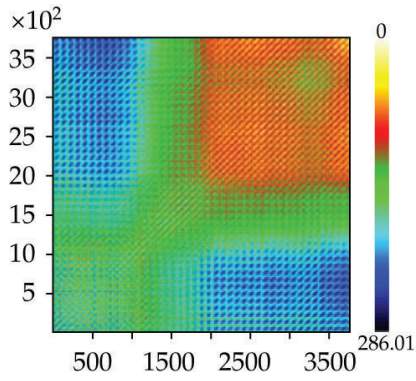

(a)

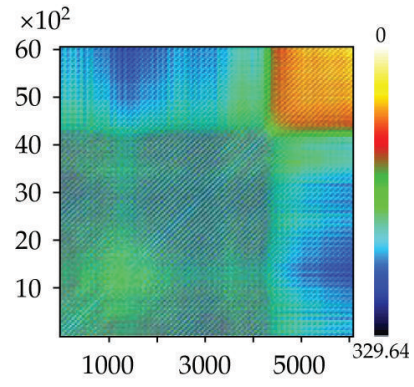

(b)

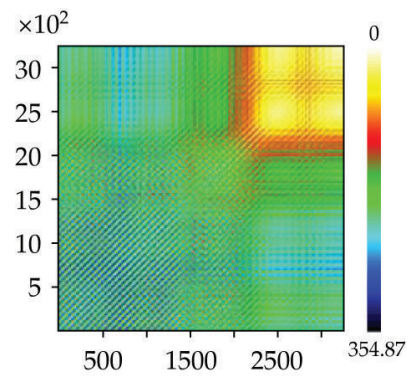

(c)

Figure 6: Recurrence plots for periodic or quasiperiodic position series.

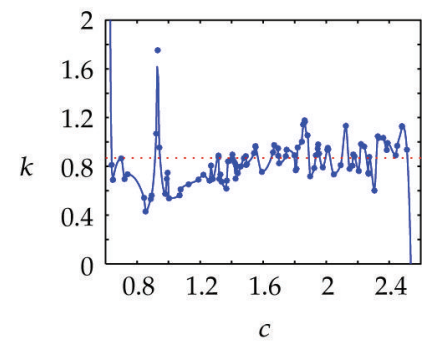

(a)

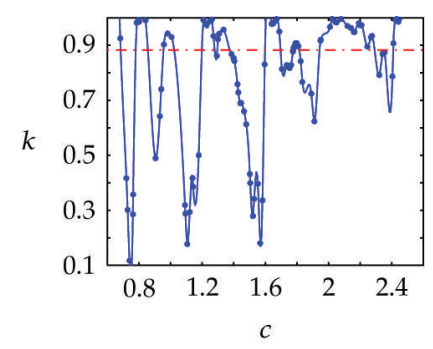

(d)

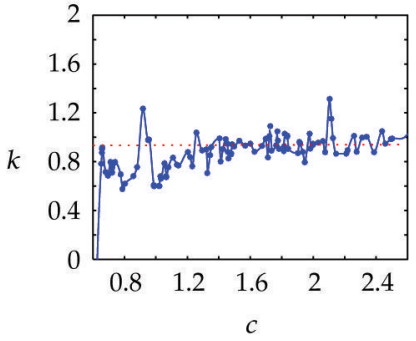

(b)

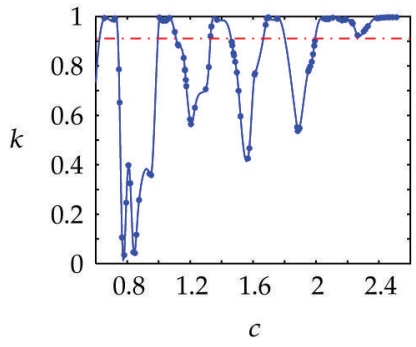

(e)

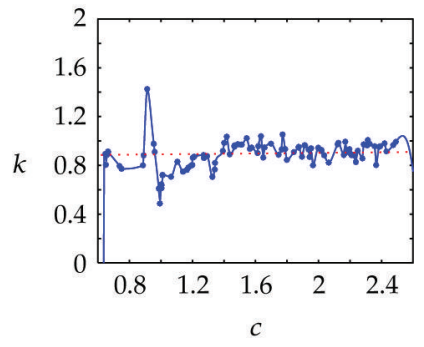

(c)

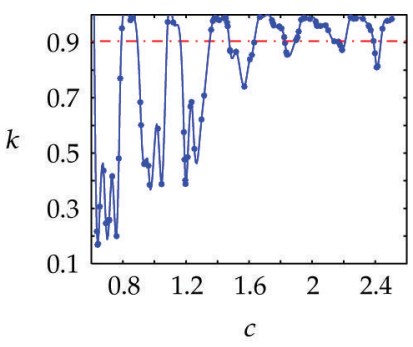

(f)

Figure 7: $k_{c}$ in the case of chaotic position series using the regression method (top) and correlation method (bottom): (a) $k=0.8626$; (b) $k=0.9542$; (c) $k=0.8916$; (d) $k=0.8942, Q 1=20 \mathrm{~kg} / \mathrm{h}, Q 2=232 \mathrm{~m}^{3} / \mathrm{h}$; (e) $k=0.9372, Q 1=21 \mathrm{~kg} / \mathrm{h}, Q 2=250 \mathrm{~m}^{3} / \mathrm{h} ;(\mathrm{f}) k=0.9046, Q 1=30 \mathrm{~kg} / \mathrm{h}, \mathrm{Q} 2=350 \mathrm{~m}^{3} / \mathrm{h}$. Red dashed lines in (a)-(f) stand for the median $k$ of $k_{c}$.

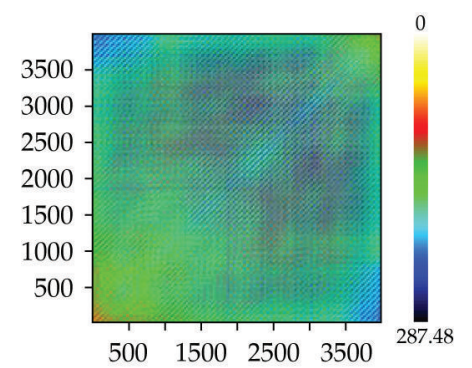

(a)

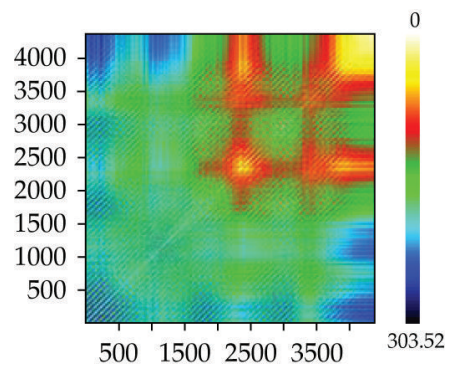

(b)

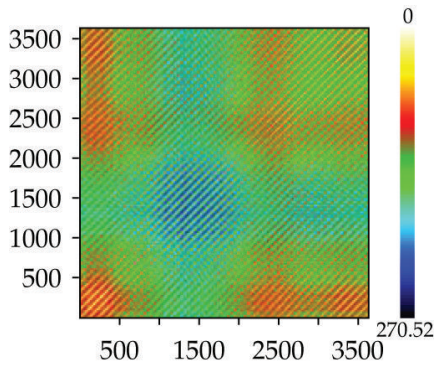

(c)

Figure 8: Recurrence plots for chaotic position series. 


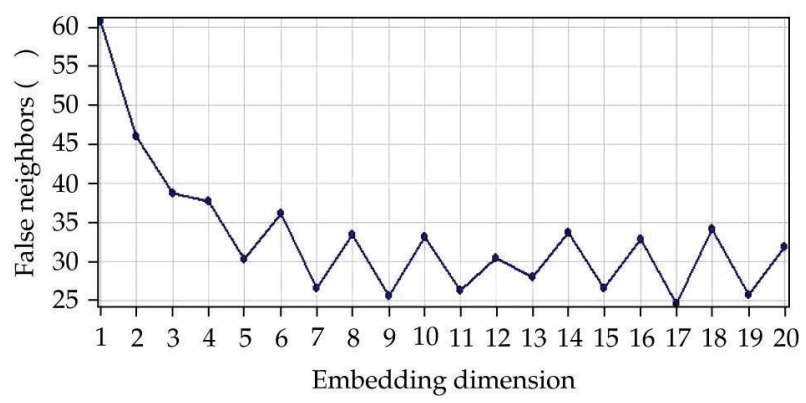

(a)

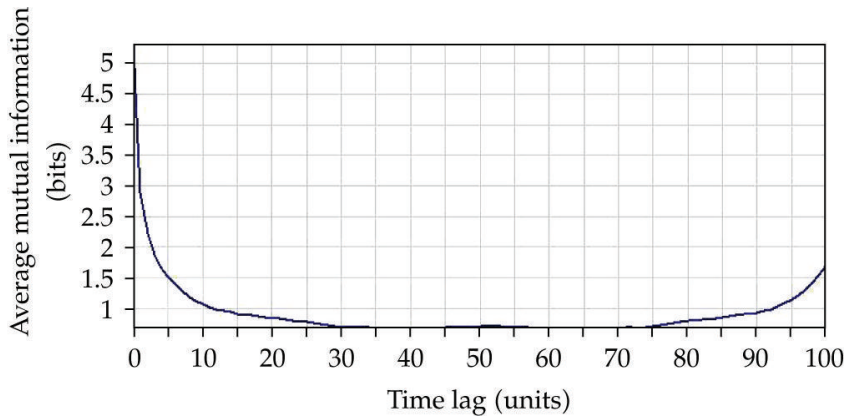

(b)

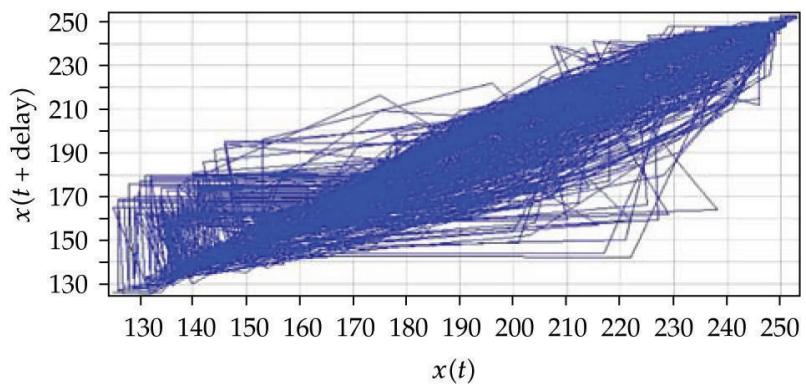

(c)

Figure 9: Percentage of false nearest neighbors (FNNs) versus the embedding dimension (a); average mutual information versus the time lag (b); reconstructed phase space (c).

Figure $9(\mathrm{c})$ only shows the projection on subspace $(x(t), x(t+$ delay $))$ of the trajectory in reconstructed phase space $(x(t), x(t+1), \ldots, x(t+$ delay $))$.

In Figure 10, a practical method [15] is used to calculate the maximum Lyapunov exponent; the maximum Lyapunov exponent is easily and accurately calculated using a leastsquares fit to the "average" line defined by plot of $\ln$ (divergence) versus time. This method is easy to implement and fast because it uses a simple measure of exponential divergence. The dotted line has a slope equal to the theoretical value of maximum Lyapunov exponent; the clear presence of a positive slope affords one with the qualitative confirmation of a positive exponent.

When combustion instabilities occur, median value $k$ of $k_{c}$ calculated for position series obtained from the flame spatial patterns begins to fluctuate between zero and one; the spatial patterns of flame in the combustion dynamical system follows a route to chaotic 


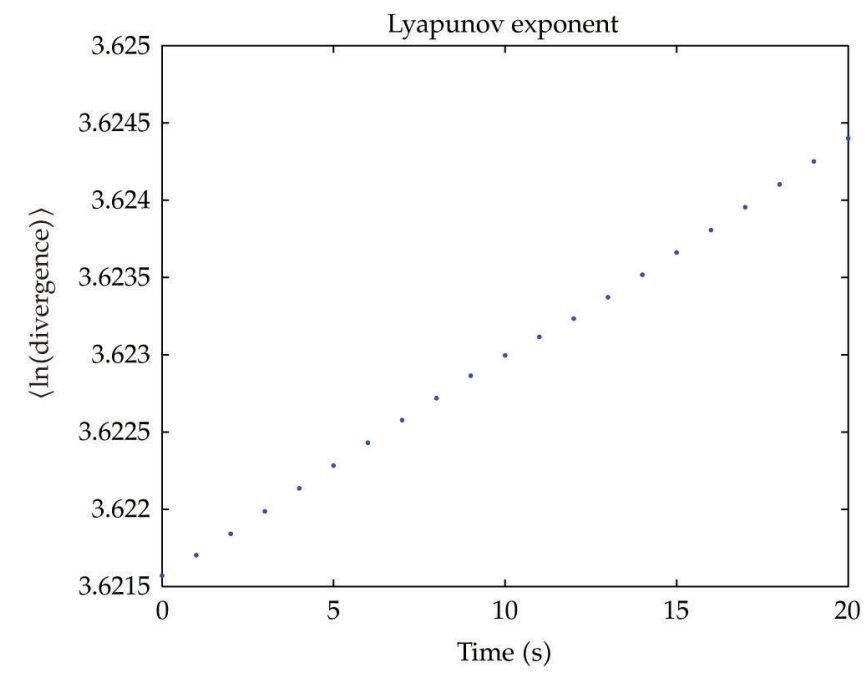

Figure 10: Lyapunov exponent profile for the chaotic position series.

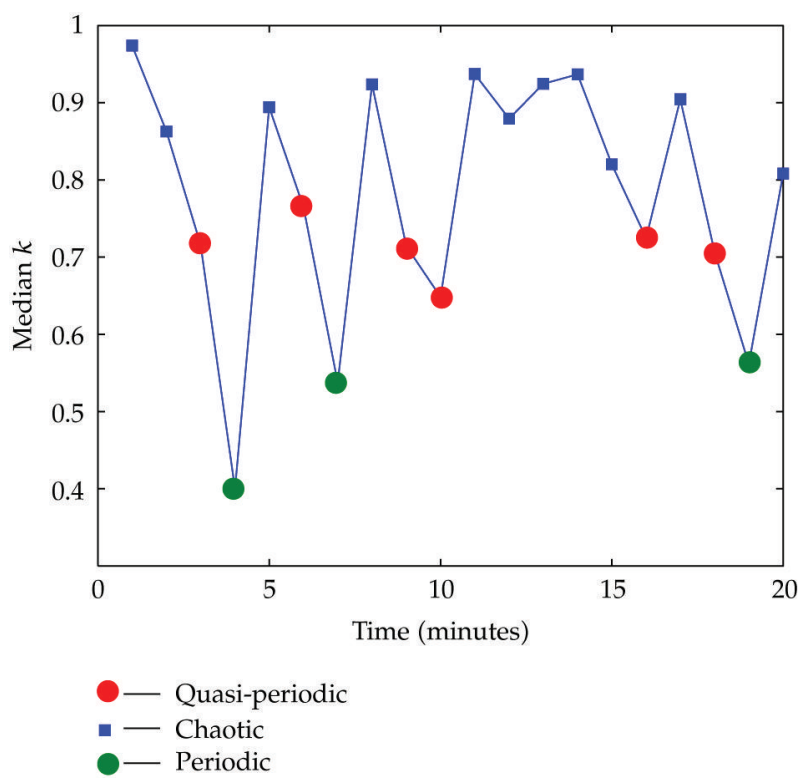

Figure 11: Evolution curve of the median of $k_{c}$ of flame position series.

spatial patterns via periodic and quasiperiodic spatial patterns. Figure 11 clearly verifies this result found in our study.

\section{Conclusion}

Unsteady motions of a nonpremixed biodiesel/air flame tip are experimentally investigated from the viewpoint of nonlinear dynamics. The fuel flow rate is varied from 15 to $30 \mathrm{~kg} / \mathrm{h}$, atomization air flow rate is set to a specific value from 0.15 to $0.4 \mathrm{~m}^{3} / \mathrm{min}$, and combustion 
air flow rate is varied from 150 to $750 \mathrm{~m}^{3} / \mathrm{h}$. The proposed methodology, based both on concepts of nonlinear dynamics and image analysis and supported by time series analysis allowed the characterization of the flame instability related to measurements based on the flame light emission. Structures of flame tip are analyzed using new image analysis technique procedures that extract position series which are representative of the relative change in temperature of combustion chamber. Chaos existence is analytically proved in odd and even numbers sequences by means of the classic method of calculating the maximum Lyapunov exponent and the 0-1 test for chaos. However, by the 0-1 test and Poincare map, chaotic, periodic, and quasiperiodic flame structures are characterized. These results indicate that the motion of the flame tip follows a route to chaos via periodic and quasiperiodic states. When the proportion between the fuel flow rate and combustion air flow is approximately controlled at $1: 12$, the combustion is relatively stable, and the positional series exhibit chaotic motion. The present work also shows that analysis based on the nonlinear dynamics, such as the $0-1$ test for chaos and Poincaré section, is valid for quantifying flame instability.

The proposed approach represents a further step towards the design of a suitable control ensuring the stability of the combustion system, which represents one of the most stringent requirements for the improvement of combustion performance and the reduction of pollutant emissions.

\section{Acknowledgments}

The authors wish to especially thank the referees for numerous detailed questions and comments that greatly improved the presentation. The authors also thank very much Professor Li Jibin, Kunming University of Science and Technology, and Professor Didier Gonze, Université Libre de Bruxelles, for essential help.

\section{References}

[1] H. Gotoda, Y. Asano, K. H. Chuah, and G. Kushida, "Nonlinear analysis on dynamic behavior of buoyancy-induced flame oscillation under swirling flow," International Journal of Heat and Mass Transfer, vol. 52, no. 23-24, pp. 5423-5432, 2009.

[2] A. Fichera, C. Losenno, and A. Pagano, "Experimental analysis of thermo-acoustic combustion instability," Applied Energy, vol. 70, no. 2, pp. 179-191, 2001.

[3] H. Gotoda and T. Ueda, "Transition from periodic to non-periodic motion of a bunsen-type premixed flame tip with burner rotation," in Proceedings of the 29th International Symposium on Combustion Hokkaido University Sapporo Japan, pp. 1503-1509, July 2002.

[4] H. Gotoda and T. Ueda, "Orbital instability and prediction of a Bunsen flame tip motion with burner rotation," Combustion and Flame, vol. 140, no. 4, pp. 287-298, 2005.

[5] S. Lei and A. Turan, "Nonlinear/chaotic analysis, modelling and control of combustion instabilities due to vaporizing sprays," Chaos, Solitons and Fractals, vol. 42, no. 3, pp. 1766-1779, 2009.

[6] G. Gianni, G. Mariotti, E. Paganini, and S. Sello, "Characterization and diagnostics of combustion thermoacoustic instabilities using nonlinear dynamics and topological methods," in Proceedings of the uropean Combustion Meeting, 2003.

[7] G. A. Gottwald and I. Melbourne, "Testing for chaos in deterministic systems with noise," Physica D, vol. 212, no. 1-2, pp. 100-110, 2005.

[8] G. A. Gottwald and I. Melbourne, "A new test for chaos in deterministic systems," Proceedings of The Royal Society of London, vol. 460, no. 2042, pp. 603-611, 2004.

[9] G. A. Gottwald and I. Melbourne, "Comment on "reliability of the 0-1 test for chaos"," Physical Review E, vol. 77, no. 2, Article ID 028201, 2008.

[10] I. Falconer, G. A. Gottwald, I. Melbourne, and K. Wormnes, "Application of the 0-1 test for chaos to experimental data," SIAM Journal on Applied Dynamical Systems, vol. 6, no. 2, pp. 395-402, 2007. 
[11] F. Ascani et al., "Detection of low-dimensional chaos in quasi-periodic time series: the 0-1test," Tech. Rep., Santa Fe Institute Complex Systems Summer School, 2008.

[12] J. Chen, T. Y. Hsu, C. C. Chen, and Y. C. Cheng, "Monitoring combustion systems using HMM probabilistic reasoning in dynamic flame images," Applied Energy, vol. 87, no. 7, pp. 2169-2179, 2010.

[13] G. Poli and D. Perugini, "Strange attractors in magmas: evidence from lava flows," Lithos, vol. 65, no. 3-4, pp. 287-297, 2002.

[14] G. A. Gottwald and I. Melbourne, "On the implementation of the 0-1 test for chaos," SIAM Journal on Applied Dynamical Systems, vol. 8, no. 1, pp. 129-145, 2009.

[15] M. T. Rosenstein, J. J. Collins, and C. J. de Luca, "A practical method for calculating largest Lyapunov exponents from small data sets," Physica D, vol. 65, no. 1-2, pp. 117-134, 1993.

[16] J. Belaire-Franch and D. Contreras, "Recurrence plots in nonlinear time series analysis: free software," Journal of Statistical Software, vol. 7, pp. 1-18, 2002.

[17] M. B. Kennel, R. Brown, and H. D. I. Abarbanel, "Determining embedding dimension for phase-space reconstruction using a geometrical construction," Physical Review A, vol. 45, no. 6, pp. 3403-3411, 1992.

[18] D. Cafagna and G. Grassi, "Fractional-order Chua's circuit: time-domain analysis, bifurcation, chaotic behavior and test for chaos," International Journal of Bifurcation and Chaos in Applied Sciences and Engineering, vol. 18, no. 3, pp. 615-639, 2008. 


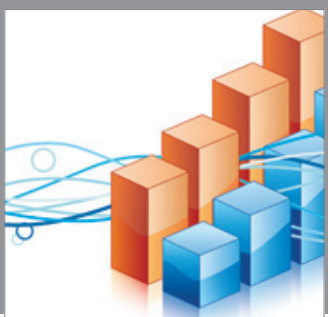

Advances in

Operations Research

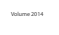

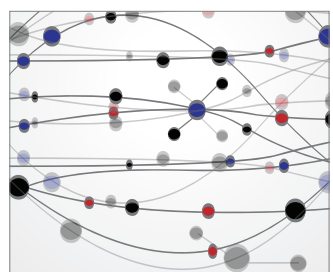

\section{The Scientific} World Journal
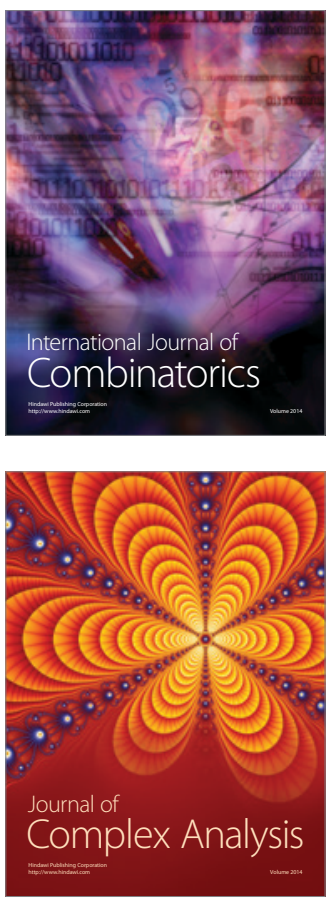

International Journal of

Mathematics and

Mathematical

Sciences
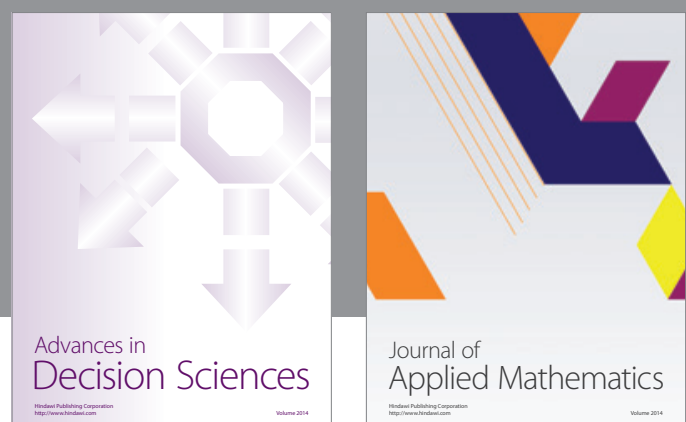

Journal of

Applied Mathematics
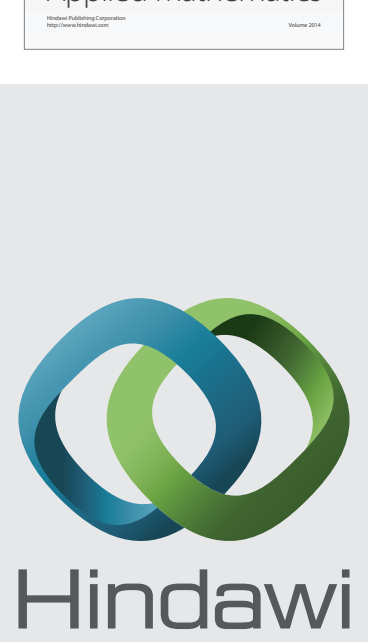

Submit your manuscripts at http://www.hindawi.com
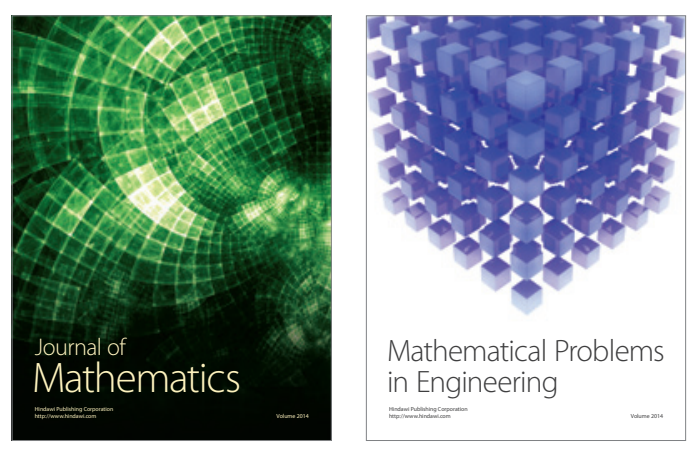

Mathematical Problems in Engineering
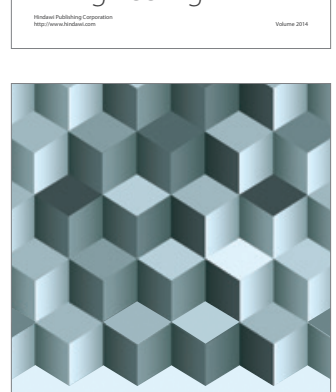

Journal of

Function Spaces
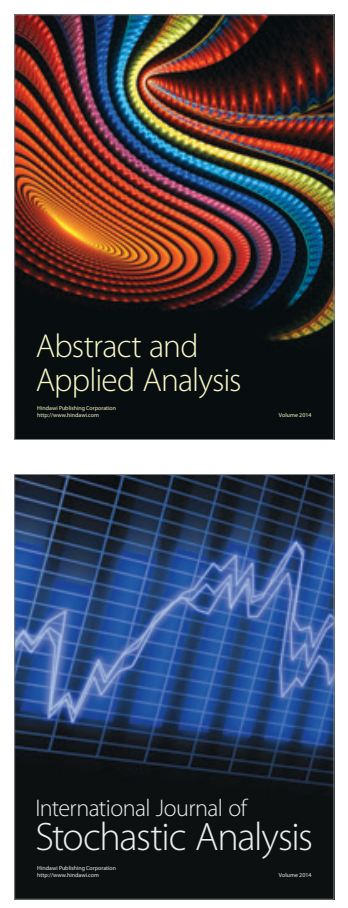

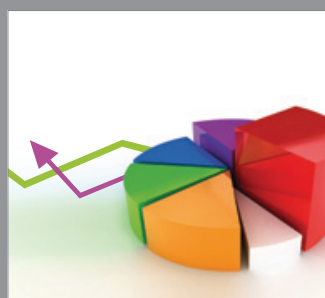

ournal of

Probability and Statistics

Promensencen
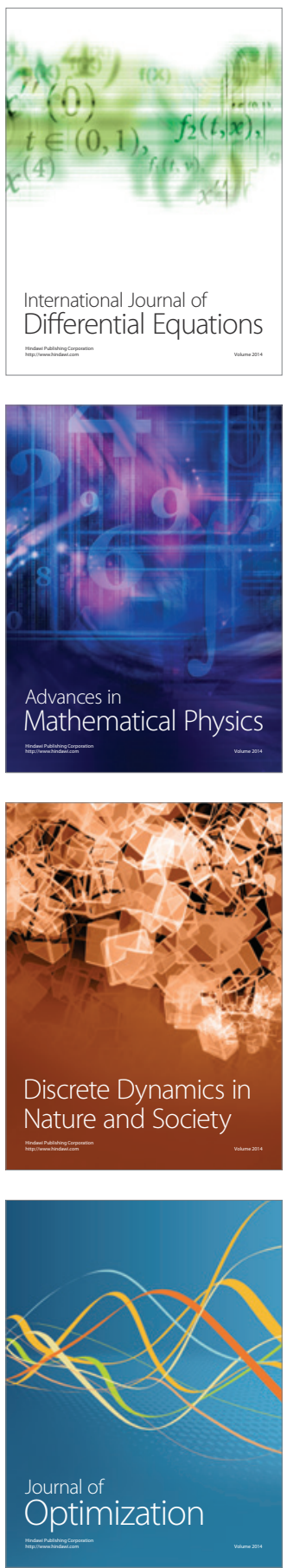